the centre of the regular tetrahedron $B_{1}, B_{2}, B_{3}, B_{4}$ of beryllium atoms. The acetate group $\mathrm{CH}_{3}-\mathrm{C}_{\mathrm{O}-}^{\mathrm{O}}$ is a plane $Y$-shaped structure, a methyl group at its foot, a carbon atom at its middle and two structurally equivalent oxygen atoms at the extremities of the arms. These are shown in the sketch at $C_{1}, C_{2}, a$ and $b$. In the 'end on' view of Fig. $2 b, B_{1} B_{2}, B_{3} B_{4}$ are the traces of the edges of the tetrahedron of beryllium atoms viewed along $X O$. These edges are parallel to 110 axes of the unit cube and lie in planes of symmetry for the holohedral crystal. The line $a b$ joining the two oxygen atoms is, at room temperatures, inclined to the line $B_{1} B_{2}$ (and $B_{3} B_{4}$ ); as the temperature rises, its mean position must be such as to produce a symmetrical distribution about this line. This can occur in several ways; for example: (I) if the atomic vibrations carry $a b$ to $a^{\prime} b^{\prime}$ (symmetry about $B_{3} B_{4}$ is secured automatically if it is present about $B_{1} B_{2}$ ), (2) if the acetate $Y$ rotates about its stem so that $a b$ in Fig. $2 b$ describe a circle. The mean position of these two atoms must have a symmetrical aspect to produce the photograph of Fig. 1, and must therefore be in, or at right angles to, $B_{1} B_{2}$.

Further investigation might throw light on the amplitude of the thermal movements to which the observed change of symmetry is due : the onset of vibration across the symmetry plane should be accompanied by an abnormal rise of specific heat. Molecular rotation in nitrate crystals has been reported by Hendricks, Posnjak and Kracek ${ }^{5}$ and is accompanied by a change of symmetry such as that here recorded. It seems that if Laue photographs are used to determine symmetry, they should be taken at as low a temperature as possible.

We are indebted to Dr. D. V. N. Hardy, Chemical Research Laboratory, for the preparation of the single crystals of basic beryllium acetate used in this investigation.

The work described above has been carried out as part of the research programme of the National Physical Laboratory, and this paper is published by permission of the Director of the Laboratory.

$$
\text { G. D. Preston. }
$$

J. TrotTer.

Metallurgy Department,

National Physical Laboratory, Teddington.

Jan. 11.

${ }^{1}$ Charlesby, Finch and Wilman, Proc. Phys. Soc., 51, 479 (1939).

'Bragg and Morgan, Proc. Roy. Soc., A, 104, 437 (1923).

'Morgan and Astbury, Proc. Roy. Soc., A, 112, 441 (1926).

- Pauling and Sherman, Proc. Nat. Acad. Sci., 20, 431 (1934).

${ }^{5}$ Kracek, Hendricks and Posnjak, Nature, 128, 411 (1931).

\section{Magnetization of Matter by Ultra-Violet Radiation}

Ehrenhaft and Banet have reported ${ }^{1}$ finding that magnetic poles are produced in various pieces of "non-magnetic" and annealed pieces of iron by exposure to ultra-violet radiation. The detecting instrument used was a simple compass needle. Focken ${ }^{2}$, on the other hand, repeated these experiments with more sensitive apparatus, but found no evidence of any such induced magnetization. We have recently attempted similar experiments.
The deflexion magnetometer used was of the reflecting type and its sensitivity was $9,800 \mathrm{~mm}$, at one metre per oersted, as compared with $9,000 \mathrm{~mm}$. at one metre for the instrument used by Focken. We consider that for specimens distant $10 \mathrm{~cm}$. from the needle, a change in the magnetic moment of 0.25 c.q.s. unit could be detected with certainty. The ultraviolet source was a D.c. mercury arc enclosed in a quartz envelope. The specimen under test was placed with its centre $10 \mathrm{~cm}$. from the needle and in the end-on position with respect to it. In this position, one surface of the specimen was fully exposed to radiation propagated at right angles to its length. Each specimen was also exposed to radiation propagated approximately parallel to its length, and to do this conveniently, each was moved to the broadside position. In these positions, the material was always placed at right angles to the magnetic meridian. With the arc running, and the specimen in place, but shielded from the radiation by a shutter, the magnetometer reading was noted. The shutter was then opened, the light spot from the magnetometer being kept under observation. This procedure ensures that the only change in conditions is exposure to radiation, other magnetizing influences such as the fields of the arc and the earth remaining substantially constant throughout any given test.

The experiments were also repeated for end-on and broadside positions when the radiation was concentrated on the specimen by means of a quartz lens.

Three types of materials in the form of rods, approximately $8 \mathrm{~cm}$. long, were tried, namely, transformer iron, mild steel and a hard steel.

In no case could any change be detected in the magnetic condition of the rods, either at the instant of exposure or after exposures ranging from $\frac{1}{2}$ hour to 3 hours. By placing the rods in a vertical position and illuminating as before, we tested for possible magnetization at right angles to the length. Here, also, the results were negative.

S. L. Martin.

Physics Laboratories,

A. K. Connor.

Melbourne Technical College, Australia.

Nov. 17.

1 Ehrenhaft, F., and Banet, L., NATURE, 147, 297 (1941).

' Focken, C. M., NATURE, 148, 438 (1941).

\section{A New Band Spectrum Associated with Nitrogen}

DURING an attempt to improve measurements on the Vegard-Kaplan bands of $\mathrm{N}_{2}$ using a silent. (ozonizer type) discharge through nitrogen as source, as recommended by Wulf and Melvin ${ }^{1}$, three new bands in the blue region of the spectrum were observed. These bands were afterwards obtained quite readily in an ordinary induction-coil discharge through nitrogen at relatively very high pressure (around $10 \mathrm{~cm}$. mercury). The bands were obtained with commercial nitrogen, with special oxygen-free nitrogen, and with atmospheric nitrogen made by bubbling air through alkaline pyrogallol. The bands do not appear at lower pressure or in discharges through air. The bands disappear if a little oxygen is mixed with the nitrogen, and hydrogen and water vapour also have an inhibiting action. In all the 